EPJ Web of Conferences 71, 00126 (2014)

DOI: $10.1051 /$ epjconf/20147100126

(C) Owned by the authors, published by EDP Sciences, 2014

\title{
Real-time Detection of Antihydrogen Annihilations and Applica- tions to Spectroscopy
}

\author{
Simone Stracka ${ }^{1, a}$ \\ ${ }^{1}$ Scuola Normale Superiore, Pisa, Italy
}

\begin{abstract}
A detection scheme based on real-time measurement of antihydrogen annihilations during radiation injection is presented, which allows an efficient use of the trapped atoms for laser and microwave spectroscopy. The application of real-time detection of $\bar{H}$ annihilations to microwave spectroscopy, which yielded the first evidence of microwaveinduced spin-flip transitions in trapped antihydrogen [1], is reported.
\end{abstract}

\section{Introduction}

Since 1980, CERN has conducted a low-energy antiproton program for precision CPT and gravity measurements, first with the Low Energy Antiproton Ring (LEAR) [2] and then with the Antiproton Decelerator (AD) [3]; a recent summary of the status of the experiments at the AD is provided, e.g., in Ref. [4]. A substantial part of the AD program is aimed to measure the energy-level structure of antihydrogen. Antihydrogen is considered one of the best systems for sensitive tests of CPT symmetry, due to the availability of precise spectroscopic techniques and of a well-understood (both experimentally and theoretically) matter-counterpart for comparison. Two-photon $1 S-2 S$ laser spectroscopy and microwave hyperfine spectroscopy have the potential to improve on existing CPT limits and, when combined with independent measurements of the static properties of its constituents (Table 1), probe the internal structure of the antiproton $[5,6]$.

One of the most apparent differences between hydrogen and antihydrogen spectroscopy is that antihydrogen must first be produced in a state suitable for spectroscopy. The four antihydrogen experiments at the AD are adopting, to this purpose, different approaches: while ASACUSA and AEgIS are working towards the production of a slow $\bar{H}$ beam, in ALPHA and ATRAP the antihydrogen produced by three-body recombination is confined in a magnetic trap with laser and microwave access [7-9]. Since manipulation of low-energy anti-matter is likely to introduce expensive losses, the production rates obtained so far are low, and only recently the ALPHA experiment succeeded in observing microwave-induced spin-flip transitions in ground-state anti-atoms [1], thus demonstrating that spectroscopy of small samples of antihydrogen is technically feasible. The small number of trapped atoms represents one of the main challenges for high precision spectroscopy, and most of the detection methods routinely used in hydrogen spectroscopy are impractical when applied to antihydrogen due to low efficiencies and difficult optical access. In the following I'll provide a brief account of the first observation of resonant quantum transitions in trapped antihydrogen and describe a possible detection scheme for laser and microwave spectroscopy in ALPHA.

ae-mail: simone.stracka@cern.ch 
Table 1. Experimental limits for some comparisons of the static properties of particles and antiparticles.

\begin{tabular}{lll}
\hline Quantity & Value & Reference \\
\hline$\left|m\left(e^{+}\right)-m\left(e^{-}\right)\right| / m_{e}$ & $<8 \times 10^{-9}$ & {$[10]$} \\
$\left|q\left(e^{+}\right)-q\left(e^{-}\right)\right| / e$ & $<2.5 \times 10^{-8}$ & {$[10,11]$} \\
{$\left[g\left(e^{+}\right)-g\left(e^{-}\right)\right] / g_{e}$} & $(-0.5 \pm 2.1) \times 10^{-12}$ & {$[12]$} \\
$|m(p)-m(\bar{p})| / m_{p}$ & $<7 \times 10^{-10}$ & {$[13,14]$} \\
$|q(p)-q(\bar{p})| / e$ & $<7 \times 10^{-10}$ & {$[13,14]$} \\
$|\mu(p)-\mu(\bar{p})| / \mu_{p}$ & $<5 \times 10^{-6}$ & {$[15]$} \\
\hline
\end{tabular}

\section{RF-induced positron spin resonance transitions in trapped antihydrogen}

The core of the ALPHA apparatus consists of a Malmberg-Penning trap in a $1 \mathrm{~T}$ solenoidal $B$ field for trapping and manipulation of charged particles, and a superconducting Ioffe-Pritchard trap for the confinement of neutral atoms [7]. At each experimental cycle, the antiprotons from the AD extraction line are slowed down in an aluminum degrader, trapped within the Penning-trap electrodes and cooled down by a combination of sympathetic cooling with an electron plasma and evaporative cooling, while positrons emitted by a ${ }^{22} \mathrm{Na}$ source and stored in a Surko-type positron accumulator are loaded into the trap. The Ioffe-Pritchard trap magnets are then energized and the cold plasmas of antiprotons $\left(\approx 10^{4} \bar{p}\right)$ and positrons $\left(\approx 10^{7} e^{+}\right)$, prepared in nested double-well potential barriers, are gently mixed for $1 \mathrm{~s}$ near the magnetic minimum, where antihydrogen is formed by three-body recombination [16]. Although the resulting antihydrogen atoms are in a highly excited state, they are expected to cascade to ground state in about $0.5 \mathrm{~s}$ [17]. A small fraction of the antiatoms are produced with a sufficiently low energy and, provided they are in a "low-field seeking" hyperfine state, are trapped in the $0.54 \mathrm{~K}$ magnetic potential well, $U=-\vec{\mu} \cdot \vec{B}$. The remaining charged particles are then cleared by the application of electric fields. After synthesis, antihydrogen can be held in the trap for several minutes for spectroscopy, and confinement times as long as $1000 \mathrm{~s}$ have been demonstrated [8].

Following ATHENA [18], ALPHA adopted a silicon vertex detector as the technology of choice for the detection of the charged products of the annihilation of the antihydrogen constituents with the surrounding matter environment. The presence of antihydrogen in the trap is signaled by a delayed antiproton annihilation signature following the intentional release of confined antihydrogen [19], accomplished by quickly lowering the walls of the magnetic trap (usually resulting in a quench of the magnets). The observation window, determined by the escape time of the antihydrogen from the trap volume, can be limited to a few tens of milliseconds around the time of antihydrogen release, thus suppressing the cosmic ray background. Since no attempt is performed to detect the positron annihilation products, irreducible background - due to, e.g., annihilations of accidentally trapped bare antiprotons - must be estimated by comparison with dedicated control experiments, such as production sequences in which the synthesis of antihydrogen is suppressed by heating the positron plasma $[1,20]$.

The production and trapping rate is modest (about one $\bar{H}$ is detected every three experimental cycles) and, so far, typically stable only over a timescale of $1-2$ weeks [7]. The goal of the measurement reported in Ref. [1] was to demonstrate the ability to conduct spectroscopy with few anti-atoms, relying on long confinement times (which allow for longer irradiation times) and coping with the existing level of reproducibility of $\bar{H}$ production rates, and apparatus and environment conditions. A suitable class of transitions for this proof-of-principle experiment was identified in the positron spin resonance (PSR) of ground-state antihydrogen, consisting of the two transitions from the "low-fieldseeking" (trappable) hyperfine states $|c\rangle$ and $|d\rangle$ to the "high-field-seeking" (untrappable) states $|b\rangle$ and $|a\rangle$, respectively (Fig. 1). 


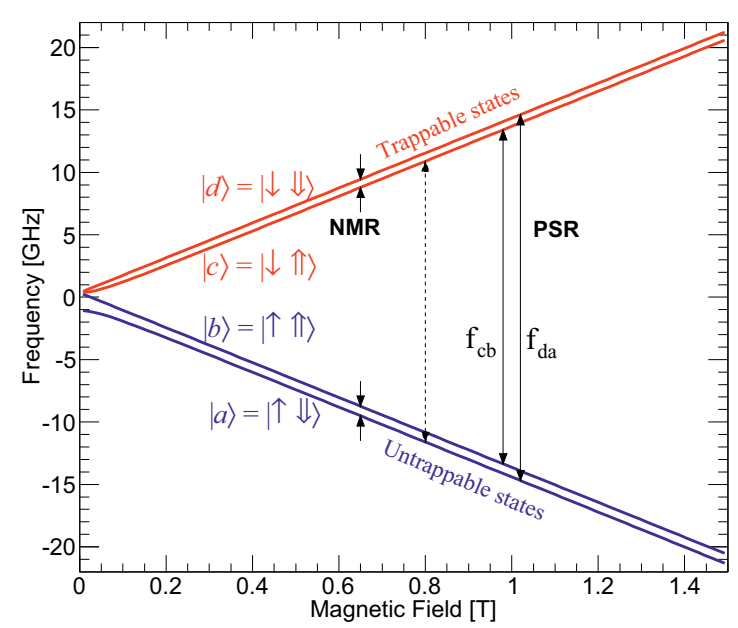

Figure 1. Diagram of the relative hyperfine energy levels of ground state antihydrogen in a magnetic field. The single arrow in the state vectors refers to the positron spin and the double arrow refers to the antiproton spin.

After holding the produced antihydrogen for $60 \mathrm{~s}$, the transitions were excited by injecting microwave radiation down the axis of the apparatus via a waveguide-fed horn antenna. Since the probability for each transition as a function of frequency has an abrupt onset at the value associated to the magnetic field minimum of the trap, a microwave frequency above but close to the onset frequency is chosen to maximize the spin-flip probability (on-resonance condition). Each $30 \mathrm{~s}$ irradiation cycle consists of two $15 \mathrm{MHz}$ frequency scans, separated by $1420.4 \mathrm{MHz}$, around the onset frequencies for the $b-c$ and $a-d$ transitions. After spin-flip the antihydrogen atom, now in a high-field-seeking state, escapes from the trap and annihilates on the electrodes. The irradiation cycle is repeated 6 times before releasing and counting the surviving antihydrogen atoms, for a total confinement time of $240 \mathrm{~s}$.

The occurrence of PSR transitions is signaled by a significant decrease in the counts of surviving antiatoms in on-resonance runs, with respect to off-resonance control experiments in which the microwave frequencies are well below the transition onset frequency and thus cannot drive the spin-flips. Such an approach is dubbed "disappearance measurement" [1]. In order to determine the frequencies corresponding to on-resonance and off-resonance conditions, the cyclotron resonance of an electron plasma (Electron Cyclotron Resonance, ECR) is used as an in-situ magnetic field diagnostics [21, 22]. This technique allows to characterize the magnetic field stability near the trap center with a $2 \mathrm{MHz}$ run-by-run reproducibility. The measurement of the absolute value of the background (solenoid) magnetic field is found to be accurate to $\pm 10 \mathrm{MHz}$ ( 3 Gauss). For the highly in-homogeneous magnetic fields of the full trap, an order of magnitude estimate of the systematic uncertainty in the determination of the minimum $B$ field is provided by the $40 \mathrm{MHz}$ discrepancy between the ECR measurement and the magnetic field model of the full trap [22]. To account for this systematic uncertainty the off-resonance condition was chosen to correspond to a $100 \mathrm{MHz}$ shift in microwave frequency with respect to the on-resonance condition [1].

The "disappearance measurement" approach critically depends on the reproducibility of experimental conditions that could affect, e.g., the antihydrogen synthesis rate, the $\bar{H}$ annihilation or escape rate during confinement, and the detection efficiency. To decouple as much as possible from these effects, on-resonance runs must be closely interspersed (on a back-to-back basis) with off-resonance and no-microwave runs. In 100 runs without microwave injection, 40 antihydrogen annihilations are observed upon lowering of the trap after $240 \mathrm{~s}$, while $23 \bar{H}$ are detected in 110 off-resonance microwave experiments. The count drops to 2 in 103 for runs in which on-resonance microwaves are injected, thus providing a preliminary indication of PSR transitions [1]. 


\section{Real-time annihilation detection for $\bar{H}$ microwave spectroscopy}

From the preceding discussion, it is evident that a "disappearance measurement" does not allow to confidently rule out sources of antihydrogen-rate decrease other than spin-flips, such as annihilations on the residual gas desorbed from the electrodes due to microwave-induced heating. One of the main disadvantages of "disappearance measurements" is that they do not allow to use the full information contained in the clear signature offered, instead, by real-time observation of antihydrogen annihilations. During the time in which the magnetic trap is energized and microwave radiation is applied, the time and the axial and radial distributions of the annihilation vertices are in fact sensitive to the mechanism responsible for their release from the trap. In the remaining of this section, the real-time observation of antihydrogen annihilations is presented, and its possible advantages are discussed.

\subsection{Event selection for real-time study of RF-induced antihydrogen annihilations}

From simulations of on-resonance experiments, most $\bar{H}$ atoms are expected to undergo a spin-flip transition and be expelled from the trap within the first few seconds of irradiation [1]. It is therefore possible to restrict the study of events collected during microwave injection to the first $30 \mathrm{~s}$ irradiation cycle of each run ( $15 \mathrm{~s}$ per transition). Integrating over 100 runs this would correspond to a $3000 \mathrm{~s}$ observation time (100 is the number of on-resonance runs that is expected to be required in order to be sensitive to spin-flip transitions, in the "disappearance" approach).

The candidate selection requirements detailed in Ref. [7, 19] would retain about $47 \mathrm{mHz}$ of cosmic ray events, corresponding to $\sim 140$ background counts, to be compared with an expected signal yield $S=30$. It is therefore apparent that the selection proposed in Ref. [7, 19] is inadequate to the realtime study of antihydrogen annihilations in spectroscopy experiments with limited statistics. A higher signal purity would also make the interpretation of the experimental distributions easier.

A more effective cosmic-background rejection was therefore designed, based on a bagged decision tree $(B D T)$ in the random forest approach [23-25], which combines nine input variables describing the position of the reconstructed annihilation vertices, the hit and track multiplicities, and topological variables characterizing the shape of the event and its orientation relative to the apparatus [1]. Observables that exhibit correlations with the reconstructed axial position $z$ of the annihilation vertices or with the event time (used as a control variables) are excluded from the set of BDT input variables. The multivariate classifier is trained to select annihilation events against cosmic ray events, using hot antihydrogen annihilations recorded during $\bar{H}$ synthesis as the signal training sample and cosmic ray events collected during dedicated runs as the background training sample. The cosmic-ray contamination of the signal training sample is at the $O(1 \%)$ level.

The selection on the BDT output, shown in Fig. 2, is optimized by maximizing the figure of merit $\epsilon_{S} /\left(N_{\sigma} / 2+\sqrt{N_{B}}\right)$ [26], where $N_{\sigma}$ is the sigma level of significance, taken here to be 3 , and $\epsilon_{S}$ and $N_{B}$ are the signal efficiency and the expected number of cosmic background events over $3000 \mathrm{~s}$, respectively. An annihilation candidate is retained if $B D T>0.90$. Compared to the default selection criteria [19], the $B D T$ based selection results in a ten-fold suppression of the cosmic rate, while retaining $\sim 75 \%$ of the signal.

\subsection{Results of the real-time measurement and further applications}

The $z$ distribution of annihilation vertices observed in real-time is expected to be highly localized around the trap center, in contrast with the broad $z$ distribution (roughly extending over a $30 \mathrm{~cm}$-wide range) observed for $\bar{H}$ atoms released when quenching the magnets. Based on simulations of the axial position $z$ of the annihilations of spin-flipped antihydrogen relative to the center of the trap, it 

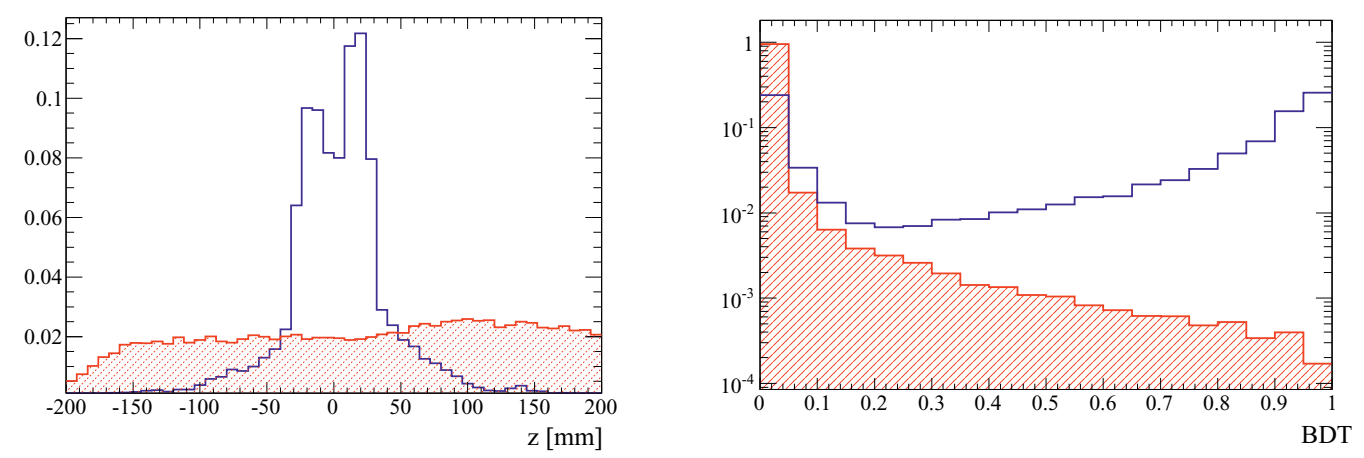

Figure 2. Distribution in the z-position of the annihilation candidate and in the trained $B D T$ output value of the training samples for background (hatched histogram) and signal (open histogram).

is therefore possible to apply a loose selection $|z|<6 \mathrm{~cm}$. This requirement affects the signal only marginally and further suppresses the background by a factor of 3, resulting in an expected cosmic background rate of $(1.7 \pm 0.3) \times 10^{-3} \mathrm{~Hz}$.

The results of the real-time measurement are shown in Fig. 3: a clear enhancement is observed in the time distribution in association with the first microwave scan. The qualitative features of the $z$-distribution also shed light on the mechanism causing annihilations: they favor the spin-flip interpretation of the observed signal over the annihilation and collisional loss of trapped anti-atom in a compromised vacuum. The data-MC comparison in Fig. 3 is only qualitative and no attempt was made to apply corrections for inefficiencies, biases, and resolution smearing to the distributions, as this was beyond the scope of a search for an excess over a background estimated from auxiliary control runs.

The good achieved signal-to-noise ratio suggests that it is possible to perform a broad frequency scan to directly look for the transition onset frequencies $\left(\propto B_{\min }\right)$, using the known relation between the transition time and the injected microwave frequency. This would reduce the need of an independent determination of the minimum magnetic field and considerably simplify the experimental sequence. The smearing of the onset frequency would provide an estimate of the magnetic field reproducibility.

The possibility to perform statistical (e.g., likelihood based) background subtraction in the $z$ observable with larger samples could also be investigated, as it would allow to extract information on microwave power and detuning [27], as well as on the $\bar{H}$ temperature, from the $z-t$ distributions. A quantitative analysis would require a good understanding and simulation of the detector performances, of the global and relative alignment of the detector, of the material budget and multiple scattering process, and of the multiplicity and momentum distributions in low-energy antihydrogen annihilations. Ultimately, systematic uncertainties would arise from the little availability of suitable control samples (limited by the control on the number size, temperature, dimensions, and position of the plasmas in the experiment), and from the lack of redundancy in the detection system (which currently limits $e-\pi$ identification capabilities, particle momentum reconstruction, and reliable efficiency studies).

\subsection{Using spin-flips to release antiatoms as a substitute for magnetic trap quenches}

The clear signature observed in Fig. 3 and the antihydrogen counts after quench show that injection of on-resonance microwaves for $30 \mathrm{~s}$ is very efficient in expelling the $\bar{H}$ atoms from the trap. It would 

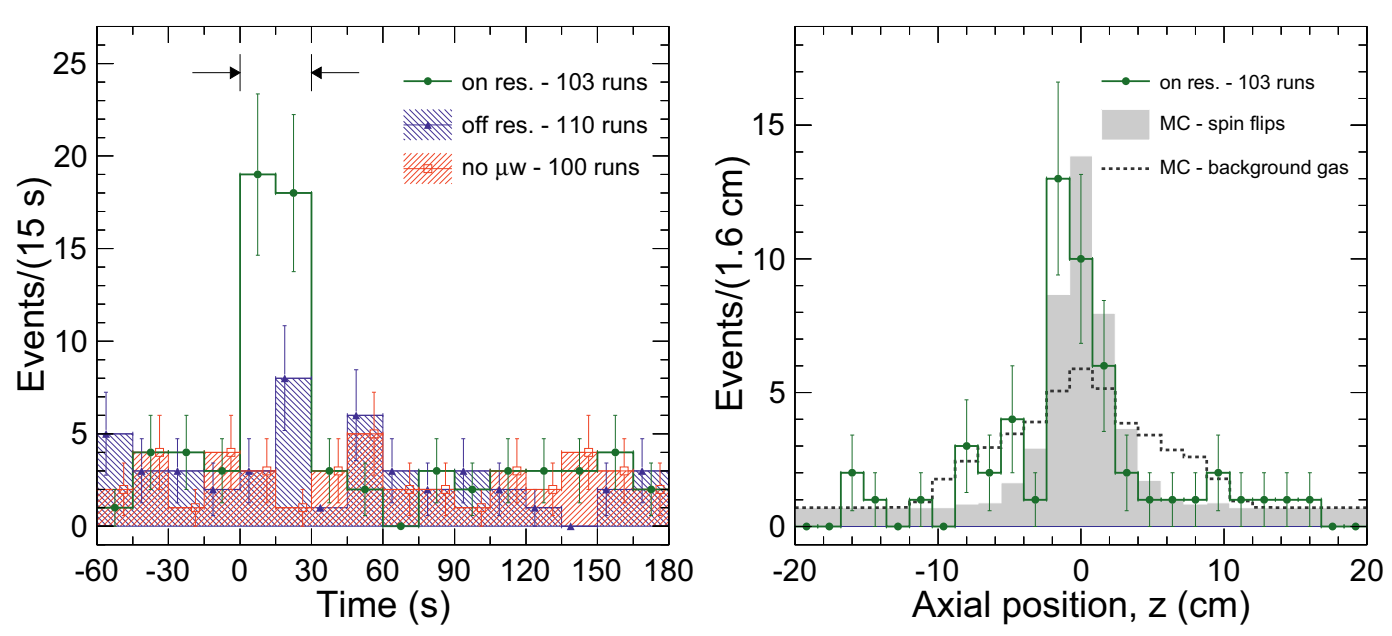

Figure 3. Left: counts as a function of time between the end of antihydrogen production $(t=-60 \mathrm{~s})$ and the trap shutdown, for $|z|<6 \mathrm{~cm}$. Microwave power is first applied at time $t=0$. Right: real-time $z$-distribution of annihilation vertices for $0<t<30 \mathrm{~s}$ in data, plotted against numerical simulations.

be therefore possible to release and count the residual antihydrogen at the end of each experiment by inducing spin-flips - via injection of high power microwaves for as long as a few tens of seconds - and applying the analysis technique described in Section 3.1.

In this way, the fast quench of the trap magnets could be avoided. It's worth noting that the antihydrogen synthesis step described in the preceding sections lasts about seven minutes, but the repetition rate of the experimental cycle is limited by the recovery interval (of the order of 15 minutes) between a quench of the magnet and the next energization of the trap: for runs with confinement times of $\lesssim 1000 \mathrm{~s}$, excluding the trap quench from the experimental sequence would increase the repetition rate significantly.

\section{Detection strategy for antihydrogen $1 S-2 S$ two-photon spectroscopy}

In hydrogen spectroscopy, a typical approach to detect the excitation to the $2 S$ level is to collect the field-induced Lyman- $\alpha$ fluorescence. Although this option was investigated for antihydrogen in Ref. [28], it was recognized that the low Lyman- $\alpha$ fluorescence collection efficiency would favor alternative methods based on the detection of the release and subsequent annihilation of excited antihydrogen atoms.

Proposed mechanisms for releasing atoms undergoing $1 S-2 S$ transitions include photo-ionization of the $2 S$ state by $243 \mathrm{~nm}$ photons [28] and the quench of the $2 S$ state to the $2 P$ state by means of an electric or microwave field, followed by a decay to a high-field-seeking state [29]. Antihydrogen $1 S-2 S$ transitions are then signaled by a decrease of annihilation counts at the end of each trial. Alternatively, the antiprotons resulting from photo-ionization can be accumulated in an electrostatic collecting well of a few eV, and then dumped to a micro-channel plate [31]. In what follows, $\tau_{\bar{H}}$ indicates the lifetime of an $\bar{H}$ atom in the trap when resonant two-photon laser radiation is injected and one of the above release mechanisms is active. 
Although the above approaches would be ideally suited for sequences featuring very long irradiation times, they are applicable only if the possible variations of the production rate and of the loss rate due to competing mechanisms (such as annihilations due to residual gas or - for the charged particles stored in a collecting well - ballistic losses) are estimated and under control. In order to perform control experiments with sufficient granularity, the duration of each experiment would preferably not exceed 4 hours (15 times longer than the longest demonstrated $\bar{H}$ confinement time [8]). An approach relying on the spin-flips and real-time analysis of the annihilations to count the residual $\bar{H}$ atoms in the trap (Sec. 3.3) would then allow to establish a $3 \sigma$ evidence of $\bar{H}$ two-photon $1 S-2 S$ transitions over a typical AD run (2000 hours of beam-time), provided $\tau_{\bar{H}} \lesssim 30000 \mathrm{~s}$. A "disappearance measurement" relying on lowering of the magnetic trap would yield similar performances.

A different detection scheme, that would allow to directly map the annihilation rate versus the laser tuning, was proposed in Ref. [29]: the antihydrogen cloud is irradiated for approximately $10 \mathrm{~ms}$, after which a short electric quench field is applied and a $10 \mathrm{~ms}$ detection window (determined by the drift time of released $\bar{H}$ atoms towards the trap wall) is opened to record any antihydrogen annihilation; the cycle is then repeated throughout the whole $\bar{H}$ confinement time. However, it should be noted that, if we estimate the electric field in the frame of the moving $\bar{H}$ atoms to be $\sim 1 \mathrm{~V} / \mathrm{cm}$, the decay rate of the $2 S$ state would be approximately $3 \mathrm{kHz}$ [32]; furthermore, $2 S$ photo-ionization by a third photon could become, at higher power, a non-negligible loss mechanism. These considerations suggest that the detection cycle proposed in Ref. [29] would be very inefficient.

These drawbacks can be circumvented by adopting, for confinement times $\lesssim 1000$ s, a real time detection approach similar to the one employed for the $\bar{H}$ spin-flip demonstration [1], where the release of $2 S$ atoms (occurring by means of any of the mechanisms described above) is monitored continuously throughout the laser injection. At the end of the irradiation period, the $\bar{H}$ atoms remaining in the trap can be counted by inducing PSR transitions. This strategy would be robust against variations in the $\bar{H}$ production rate, and the measurement of the annihilation time would allow to correlate the observed $1 S-2 S$ transitions to the associated laser detuning, with a time resolution better than the lifetime of $2 S$ atoms in the magnetic trap.

Here, the main source of background is represented by cosmic rays, which can be characterized during off-beam time. As shown previously, it is convenient to deal with localized $z$-distributions for the quenched $\bar{H}$, as this improves the rejection of cosmic background and allows to confirm the mechanism responsible for the antihydrogen release. This could relieve the need to conduct control experiments, thus effectively doubling the available sample. A localized distribution of the $\bar{H}$ signal could be obtained, e.g., by reducing the distance between the mirror coils of the Joffe-Pritchard trap, by increasing the quenching electric fields around the central region of the trap, or by using high power on-resonance microwave radiation to quench the $2 S$ state. Here we assume that a fiducial cut $|z|<10 \mathrm{~cm}$ is applied and that the $B D T$ selection is slightly tightened, resulting in a signal efficiency of $60 \%$ and a cosmic background rate of $1 \mathrm{mHz}$. This would allow, over a typical AD run, to provide evidence of $\bar{H} 1 S-2 S$ transitions for any $\tau_{\bar{H}}<4000 \mathrm{~s}$. Performances could be further improved by an ad hoc training of the multivariate classifier, but for irradiation times $\lesssim 500$ s the real-time detection already offers better sensitivity than the "disappearance" measurements.

\section{Summary}

Real-time detection of antihydrogen release in spectroscopy experiments has been presented. This approach was successfully applied to microwave spectroscopy and led to the first evidence of resonant quantum transitions in trapped antihydrogen. A "disappearance" measurement based on positron spin flips appears to be a viable alternative to a "disappearance" approach based on lowering the magnetic 
trap, and may help improving reproducibility by excluding the quenches of the magnets from the experimental sequences. It could thus find application in the forthcoming $\bar{H} 1 S-2 S$ spectroscopy programs. With sufficient statistics, the study of the $z$ and $t$ distributions for different detuning values could provide information about the temperature of the confined $\bar{H}$ and prove valuable in establishing laser cooling of antihydrogen [33]. Prospects for antihydrogen $1 S-2 S$ spectroscopy based on the realtime detection of $\bar{H}$ annihilation positions have also been presented as the most promising approach for spectroscopy with irradiation times $\lesssim 500$ s per experimental cycle.

\section{References}

[1] C. Amole et al., Nature 483, 439 (2012).

[2] P. Lefevre et al., in 11th International Conference on High-energy Accelerators, edited by W.S. Newman (Birkhauser Verlag, Basel, 1980) pp.819-823.

[3] S. Baird et al., Nucl. Instrum. Methods A 391, 210 (1997).

[4] M. Hori and J. Walz, Prog. Part. Nucl. Phys. 72, 206 (2013).

[5] K. Pachucki et al., J. Phys. B 29, 177 (1996).

[6] S. J. Brodsky et al., Phys. Rev. Lett. 94, 022001 (2005).

[7] G. B. Andresen et al., Nature 468, 673 (2010).

[8] G. B. Andresen et al., Nature Physics 7, 558 (2011).

[9] G. Gabrielse et al., Phys. Rev. Lett. 108, 113002 (2012).

[10] M. S. Fee et al., Phys. Rev. A 48, 192 (1993).

[11] J. Hughes and B. I. Deutch, Phys. Rev. Lett. 69, 578 (1992).

[12] R. S. Van Dyck et al., Phys. Rev. Lett. 59, 26 (1987).

[13] G. Gabrielse et al., Phys. Rev. Lett. 82, 3198 (1999).

[14] M. Hori et al., Nature 475, 484 (2011).

[15] J. DiSciacca et al., Phys. Rev. Lett. 110, 130801 (2013).

[16] G. Gabrielse et al., Phys. Lett. A 129, 38 (1988).

[17] T. Topçu and F. Robicheaux, Phys. Rev. A 73, 043405 (2006).

[18] M. Amoretti et al., Nucl. Instrum. Methods A 518, 679 (2004).

[19] G. B. Andresen et al., Nucl. Instrum. Methods A 684, 73 (2012).

[20] M. C. Fujiwara et al., Phys. Rev. Lett. 101, 053401 (2008).

[21] T. Friesen et al., AIP Conf. Proc. 1521, 123 (2013).

[22] C. Amole et al., to be published in New J. Phys. (2013).

[23] L. Breiman, Mach. Learn. 45, 5 (2001).

[24] I. Narsky, arXiv:physics/0507143 (2005).

[25] I. Narsky, arXiv:physics/0507157 (2005).

[26] G. Punzi, in Proceedings of the Conference on Statistical Problems in Particle Physics, Astrophysics and Cosmology (PHYSTAT2003), edited by L. Lyons (SLAC, Stanford, 2003) pp.79-83.

[27] M. D. Ashkezari, in preparation.

[28] T. W. Hänsch et al., Hyperfine Interactions 76, 457 (1993).

[29] M. H. Holzscheiter et al., Rep. Prog. Phys. 62, 1 (1999).

[30] M. H. Holzscheiter et al., Physics Reports 402, 1 (2004).

[31] M. C. Fujiwara, arXiv:hep-ex/0507082 (2005).

[32] W. L. Fite et al., Phys. Rev. 116, 363 (1959).

[33] P. H. Donnan et al., J. Phys. B 46025302 (2013). 